\title{
Multi-Sensor 3D Scanner For Postural Analysis
}

\author{
Mario Gazziro $^{1}$, Rodrigo Teiske China ${ }^{1}$, Eduardo Machado Real ${ }^{1}$, \\ Reginaldo Kisho Fukuchi ${ }^{1}$, João Paulo Gois ${ }^{1}$ \\ ${ }^{1}$ Federal University of ABC, Brazil, (UFABC) \\ \{mario.gazziro, eduardo.real, reginaldo.fukuchi, joao.gois\}@ufabc.edu.br
}

\begin{abstract}
Posture is usually defined as the relative position of body elements. The ideal posture is the one where the articulations are under a minimum amount of stress but it is never achieved due to individual factors. It is important to measure this lack of balance to minimize its effects in long term. This paper presents the creation of a multifunctional $3 D$ scanner for posture analysis that allows the measurement of distances and angles between anatomic points of the body in a semi-automatic way, adding applications that only $3 D$ information can aggregate, like the measurement of body volume to calculate the subject's body fat percentage and body part perimeters. This equipment was built using 2 RGB-D sensors combined with the reconstruction library ReconstructMe and the MeshLab software, creating an easy-to-use and interactive system. The developed application was used in postural analysis tests of a mannequin following the SAPO protocol of posture assessment and compared to the SAPO software, indicating good correspondence between the methods.
\end{abstract}

Resumo. A postura é geralmente definida como a posição relativa dos elementos do corpo. A postura ideal é aquela em que as articulações estão sob uma quantidade mínima de estresse, mas nunca são alcançadas devido a fatores individuais. É importante medir essa falta de equilíbrio para minimizar seus efeitos a longo prazo. Este artigo apresenta a criação de um scanner $3 D$ multifuncional para análise postural que permite a medição de distâncias e ângulos entre pontos anatômicos do corpo de forma semi-automática, adicionando aplicações que somente informações $3 D$ podem agregar, como a medição do volume corporal para calcular o percentual de gordura corporal e os perímetros das partes do corpo. Este equipamento foi construído usando 2 sensores $R G B-D$ combinados com a biblioteca de reconstrução ReconstructMe e ao software MeshLab, criando um sistema interativo e fácil de usar. A aplicação desenvolvida foi utilizada em testes de análise postural de um manequim seguindo o protocolo de avaliação postural SAPO e comparada com o software original SAPO (mesmo do protocolo), indicando boa correspondência entre os métodos.

\section{Introduction}

The body posture is usually defined as the relative disposition of body joints. The ideal alignment is the one in which the muscles and articulations are under minimum stress. This posture may never be achieved due to individual factors like congenital and acquired diseases, unguided and inadequate physical activities, imbalances in muscles, poor physical positioning over long periods of time, obesity and respiratory and eating disorders. It 
is important to know the level of postural imbalance in order to minimize its effects and treat them as soon as possible to improve therapeutic responses.

Postural evaluation in the orthostatic position has been widely used as a diagnostic, planning and physical therapy monitoring tool for several decades, but a universal method of postural analysis has not yet been defined. Traditionally the clinical evaluation of posture uses x-ray examinations, considered the gold standard for posture analysis. However, these tests need to be performed periodically and the effects of continued exposure to radiation can harm the patient, increasing the risk of developing several types of cancer.

Several non-radiographic techniques have been used to perform this type of analysis. One of the most common in clinics and gyms is the use of a symetrograph where the user is placed behind the device and their posture and symmetries are analyzed with respect to the lines of the equipment. This analysis, however, is considered subjective, since it depends on the experience of the evaluator, and only qualitative, indicating only the presence or not of some imbalance, without specifying its degree.

Another common method of evaluation is manual goniometry. In this type of examination, a goniometer (a linear and angular ruler) is used to evaluate angles between body positions. This method is advantageous due to the low cost of the instrument but, similarly to the use of the symetrograph, is a test of difficult repeatability due to the previous experience of the evaluators.

Among the alternatives proposed to avoid a subjective examination or that could be harmful to the patient, one of the most popular in recent years was the postural analysis by computerized photogrammetry (or digital photogrammetry), which uses digital imaging methods (digital cameras) combined with computational image processing. This type of evaluation consists of using software to mark previously determined points in patient photos, in order to calculate angles and distances between these markings, increasing the accuracy and repeatability of examinations. In recent years there has been a growing number of scientific research using this type of exam ([Asanovic et al. 2019] and [Danckaers et al. 2019]), among which we can mention the SAPO - Postural Assessment Software [Ferreira 2005], one of the most used and reliable software.

Recently, low-cost devices have been created to obtain three-dimensional information using optical technologies such as structured light and time-of-flight. These devices, along with applications that allow the reconstruction of objects, make up systems known as 3D scanners, that allow the creation of three-dimensional models of the human body in relatively short times, in a non-invasive manner and with easy repeatability of results, making it a valid option for use in postural analysis exams.

The goal of this project is to create a three-dimensional scanning equipment that can reconstruct human 3D models accurately for use in medical analysis. Using 3D scanning techniques, three-dimensional mesh processing and extraction of geometric measurements, an easy-to-use application was created for measuring posture, body fat percentage and perimeter of body sections. 


\section{Related Work}

\subsection{D Scanners}

A 3D scanner is a device for measuring elements of the physical world that creates virtual models that reproduce shape and volume proportional to the element in the real world. To perform this, the 3D scanner generates a point cloud representing a position in 3D physical space ( $x, y, z$ coordinates) that are converted into a polygonal mesh that captures the geometry of physical objects.

The first full-body scanners emerged in the late $20^{\text {th }}$ and early $21^{\text {st }}$ century, using lasers as the main component and requiring large space for its installation. With the emergence of new technologies, such as low-cost RGB-D (Red, Green, Blue - Depth) sensors for entertainment purposes, new research has emerged in the area of 3D scanners to try to reduce equipment costs and the physical space occupied by them.

The RGB-D sensors use CMOS (Complementary Metal-Oxide Semiconductor) technology to obtain color and depth information. The depth sensor captures the reflection of a known pattern emitted by an infrared light source. The internal image processor of the sensor uses the relative position of the dots present in the emission pattern to calculate the distance between the camera-laser plane and the object plane, in a technique called triangulation.

In 2012 Cui et al. [Cui et al. 2012] carried out a study for the use of a single RGB-D sensor for the scanning of an entire person, the KinectAvatar system. The system proposed the use of super resolution algorithms to overcome problems of low quality resolution and demonstrated the use of such devices for 3D scanning.

Tong et al. (2012) [Tong et al. 2012] used a different system with three sensors and a rotating base to scan a whole body, as shown in Figure 1. The advantage of using more sensors to decrease the distance between the scanned object and the cameras proved to be valid and was adopted in other studies.

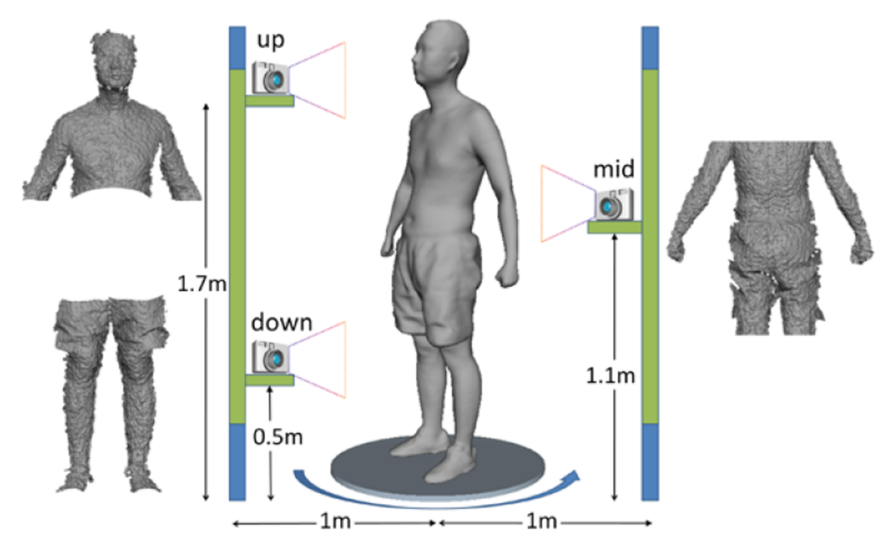

Figure 1. System with 3 RGB-D Sensors and a rotating base (extracted from [Tong et al. 2012]).

With the evolution of these studies, commercial 3D scanners using this type of sensors began to arrive in the market, each one using different techniques for the acquisition, model reconstruction and mesh processing. These new 3D scanners are used mainly 
(but not only) to monitor the physical condition of the user. Some equipment does not create a model of the scanned person, but uses the information obtained in the process to modify a generic default template. None of the current RGB-D based 3D scanners can automatically produce a colored mesh of the user.

\subsection{D Processing and Reconstruction}

The 3D scanner systems obtain data in form of 3D point clouds and needs to work with other methods to perform the alignment of these point clouds and to use them to reconstruct a polygonal model known as 3D mesh. The work of Berger et al. (2014) [Berger et al. 2014] undertakes a survey of the state of the art of three-dimensional mesh reconstruction techniques. Among these techniques one of the most used is the Poisson reconstruction algorithm, proposed in [Kazhdan et al. 2006].

For the processing of polygonal meshes the MeshLab software was created, presented in [Cignoni et al. 2008]. This free software offers several state-of-the-art threedimensional mesh processing capabilities, including the Poisson reconstruction algorithm, being widely used because of its simplicity.

\subsection{Postural Analysis Based on Computerized Photogrammetry}

For postural analysis, photogrammetry is used to perform calculations of distances and angles between anatomical points in an image of the patient, with the help of proper software for this task. The anatomical points are marked on the subject using colored adhesive paper, 3D spheres or any visually distinguishable method. Photographs of the marked subject are taken and used as input to the software for digital marking.

Several postural analysis software have been proposed over the years, but there is still no consensus as to which software is more appropriate, mainly because there is no standardization of the angles used to verify certain postural changes [Camelo et al. 2015]. In addition, for many of the existing software there are still few studies of their reliability, reproducibility and validity.

One of the most popular and studied free tools in the area is SAPO (Postural Assessment Software), created at the University of São Paulo in 2003. It was developed in Java and has as objectives, besides the postural analysis, the generation of a database with information coming from research centers and the provision of information on postural control [Ferreira 2005], and has a standardized marking of anatomical points, the so-called "SAPO Marking Protocol". Several studies have been proposed to analyze its reliability and reproducibility, concluding that it is a reliable and valid alternative to perform measurements, being compared with traditional methods of measurement and presenting good results.

The SAPO marking protocol is a suggestion of marking points and measures for postural evaluation. It suggests 3 orientations of imagery: front, back and lateral. Each orientation has its set of points and measurements, indicated in Figure 2. The list of points and measures can be found in [Duarte et al. ].

\subsection{Body Fat Estimation}

In addition to the measurement of postural deviations, other information of great importance for the physical evaluation of an individual is the measurement of the different body 


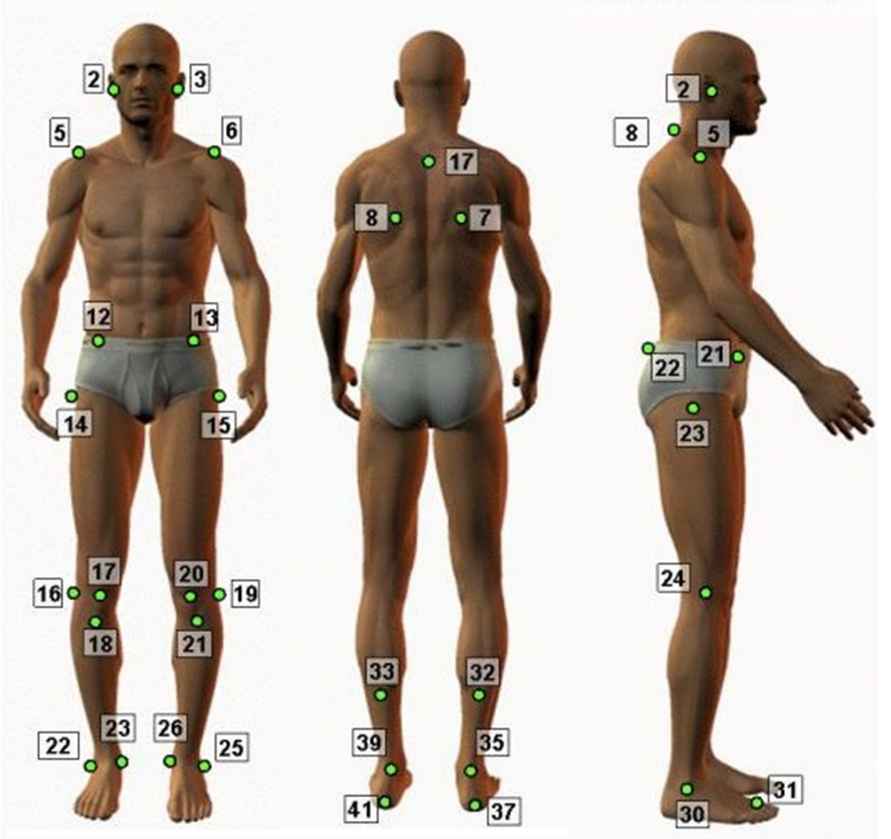

Figure 2. SAPO marking protocol (adapted from [Duarte et al. ]).

components in relation to the total body mass, especially the levels of fat and muscle mass, since they have a strict relation with the physical aptitude and health.

Delgado (2004) [Delgado 2004] reviews methods for measuring and classifying body composition. Among the classifications cited are the two-compartment model, which divides the body into two main parts: fat and fat-free. This classification was used in [Lacerda 2015], where the author implements a 3D scanner method to replace the hydrostatic weighing in the classic densitometry exam, which is then compared to bioimpedance and adipometry techniques, demonstrating the viability of the method.

\subsection{Anthropometric Measurements}

Another important application using a 3D scanner is the analysis of anthropometric measurements, which can be applied to the customization of products such as clothing and footwear or to the creation of special mechanical prostheses. Traditionally the dimensions of the human body are extracted manually through measuring tapes, but this process is time-consuming and requires direct contact with the object of study. References [Apeagyei 2010] and [Spahiu et al. 2014] are examples of 3D scanner uses for garment fitting.

Body section perimeter measurements are used to demonstrate evolution in physical size, with a strong focus on users of fitness and bodybuilding gyms.

To perform this type of analysis the 3D model needs to be segmented. The area of 3D mesh segmentation methods has been much studied since the beginning of the century. A review and analysis of some of the existing methods can be found in [Chen et al. 2009]. These methods, however, are often very complex and consume significant computational resources. For the analysis proposed in this paper, the simpler segmentations as developed in [Zhong and Xu 2006] and [Han and Nam 2011] may be more advantageous, consider- 
ing that for a medical examination, the segmentation method does not have to be pose invariant and will always be applied in human models.

\section{Methodology}

\subsection{Construction of a 3D Scanner}

The focus in this project is to use 3D meshes for the health and fitness analysis discussed in previous sections. But the available 3D scanners do not reconstruct a colored model, and for a posture analysis based on markers placed on the body the color information is vital. To perform an acquisition of a 3D model that fits the purposes of this work a structure was designed. The equipment has a turntable, a vertical physical structure assembled with aluminum profiles, plastic cover and two RGB-D sensors. The constructed scanner can be seen in Figure 3.

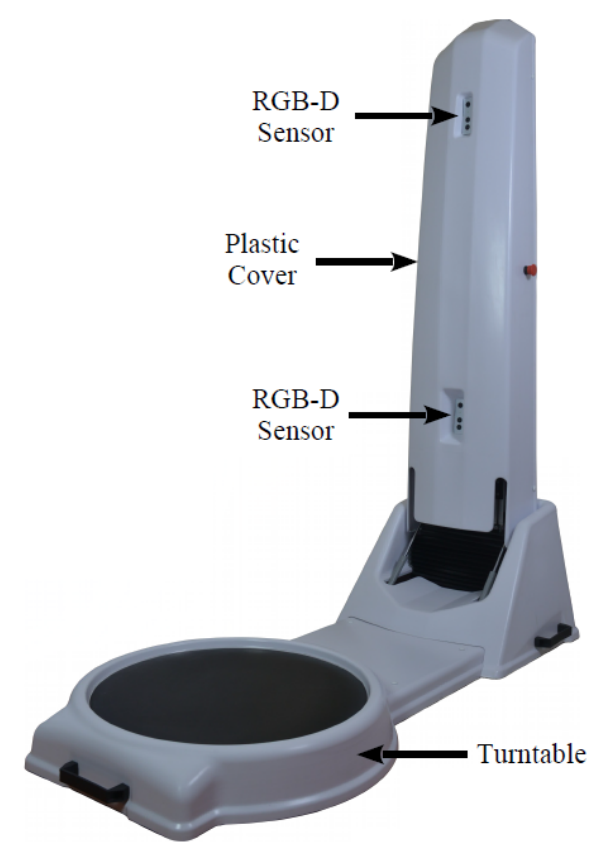

Figure 3. Multi-sensor 3D scanner.

The choice for two sensors was based on the studies reviewed in previous sections. A single sensor would require the patient to be too far away for his whole body to be scanned or else the sensor would have to be moved vertically to cover the largest possible area. This second type of system is mechanically more complex and costly. The use of more sensors is recommended, but increases the data flow that the computer must manage, requiring a machine with better performance and especially a better graphics processing unit (GPU). To decrease the required distance and to encompass a larger scan area the device is rotated at $90^{\circ}$ because of its greater field-of-view in this orientation.

The center of the turntable is located 1.10 meters away from the vertical post with the sensors and remains still until the user starts the scanning process. When the process starts the computer sends a signal to the microcontroller so that it activates its relay module, thus allowing the power supply and the operation of the turntable. The platform gently rotates the user $360^{\circ}$ in about 30 seconds while the sensors acquire depth and color information. 
The scanning software is built using the ReconstructMe [Heindl et al. 2015] SDK and manages the data of the two sensors simultaneously, creating a single 3D model at the end of the process. This model, however, contains open holes and unwanted parts and needs to be treated.

The desktop computer used to control the scanner has a NVIDIA GTX 750 graphics card (380 cuda cores), a Intel I3 CPU of $3.2 \mathrm{GHz}$ and $8 \mathrm{~GB}$ of total RAM memory.

\subsection{Mesh Processing}

The mesh processing is performed using the MeshLab software. This software allows the creation of scripts to automate the process. Two main steps need to be performed: removal of defects and unwanted parts and reconstruction of the model using the Poisson method.

The generated mesh contains unwanted parts such as the ground, for example. In order for these parts to be removed with the MeshLab software the ground must be disconnected from the main part. To allow its removal the first step to be performed is to create holes in this region so that it can be considered as a separate object. The easiest way to accomplish this step is to eliminate mesh polygons that are located below a height threshold and have approximately vertical orientation (normal vector related with horizontal ground faces). With this pre-processing done and the ground disconnected from the body we can use filters and functions of MeshLab to eliminate the rest of the unwanted sections. The following Cleaning and Repairing category filters can be used for the task:

- Remove Duplicated Faces

- Remove Duplicated Vertex

- Remove Zero Area Faces

- Remove Unreferenced Vertex

- Remove Isolated Pieces

The reconstruction of the model is done using the Poisson reconstruction function present in the Remeshing, Simplification and Reconstruction category of the MeshLab filters. As an input value, it is necessary to define the reconstruction resolution by modifying the Octree Depth parameter. As this parameter increases, higher resolution functions are used for reconstruction, capturing more accurate details, however this increases the processing time. The value of 10 presented good mesh quality. The result after the reconstruction of the model is a closed mesh (without holes), but without color. An additional step required is the colorization of the new mesh. For this, the colors associated with the vertices before reconstruction are transferred to the color array of the vertices after the reconstruction using the Vertex Attribute Transfer filter of the Sampling category.

\subsection{Postural Analysis}

The user to be scanned must wear minimal clothing so that no volume beyond the body is scanned. A swimming cap should be used to minimize the patient's hair volume. For posture examinations, the marking of anatomical points must be performed. This step follows the standard of postural analysis by photogrammetry and consists of physically marking the anatomical points of interest by means of palpation of the bone ends. For 
the tests in this project, the points defined by the SAPO protocol were considered. The chosen type of markers is composed by small circular colored self-adhesive paper, but which can be replaced by any material that is noticeably distinguishable from the rest of the model.

The marking of digital points on the mesh must be performed using mouse clicks in a previously defined order so that it is possible to relate the position of each digital marker to its corresponding physical world. To this end, a markup tracking system was created with a standard model also in 3D. With the point selected, it is saved in a list of markers and it is necessary to modify the model in some way such that the marked point becomes visible to the user of the system. For this the points within a $15 \mathrm{~mm}$ radius were painted digitally. The list of marked points on the model is used to calculate the measures described in the SAPO protocol.

\subsection{Body Fat Estimation}

The process of measuring the percentage of body fat follows the one described in [Lacerda 2015]. The volume of the mesh is calculated and used as the subject's volume. The air volume in the subject's lungs must be discounted from this value. A weighting scale is used to measure the total mass of the user and then the method estimates the body fat from the user's density.

\subsection{Body Perimeters Measurement}

The body must be segmented in 5 sections: right arm, left arm, right leg, left leg and head+torso. With the mesh already segmented it is necessary to use a horizontal cut plane to define the height where the perimeters of the body will be calculated. Each section of the body present in this cut plane should be considered separately. The perimeter of each section is calculated as the perimeter of the convex hull containing the considered section.

\subsection{Software Development}

The software was developed using the $Q t$ framework and OpenGL and GLSL functions to manage the $3 \mathrm{D}$ data. The scanning application was created using the ReconstructMe libraries available on the ReconstructMe SDK.

\section{Results}

A software was developed to perform the easy acquisition of 3D models, the automatic processing of the meshes and the health and fitness analysis described in previous sections. The posture analysis follows the SAPO protocol, but an extra functionality was added to create a new protocol of examination.

All modules have been successfully implemented and work as expected, but for this they depend on the user's position on the platform at the time of acquisition of their 3D model. If the user does not remain still the generated mesh will present errors and the volume and dimensions observed will not correspond to the real object. If the scanned pose is not correct, errors may occur in the segmentation method and, consequently, in the measurement of body perimeters. Other external factors, such as the ambient lighting where the equipment is installed, directly affect the quality of the mesh generated. The scanning software was designed to obtain information only from objects within the area 
bounded by the turntable, but a free area around the equipment is recommended to avoid acquiring data from the user's background objects, such as a wall or other people passing by.

The digital marking process allows the user to rotate the model by moving it around the screen. As the main model rotates the template model rotates together, illustrating the positioning of all the markers that must be inserted, as can be seen in Figure 4.

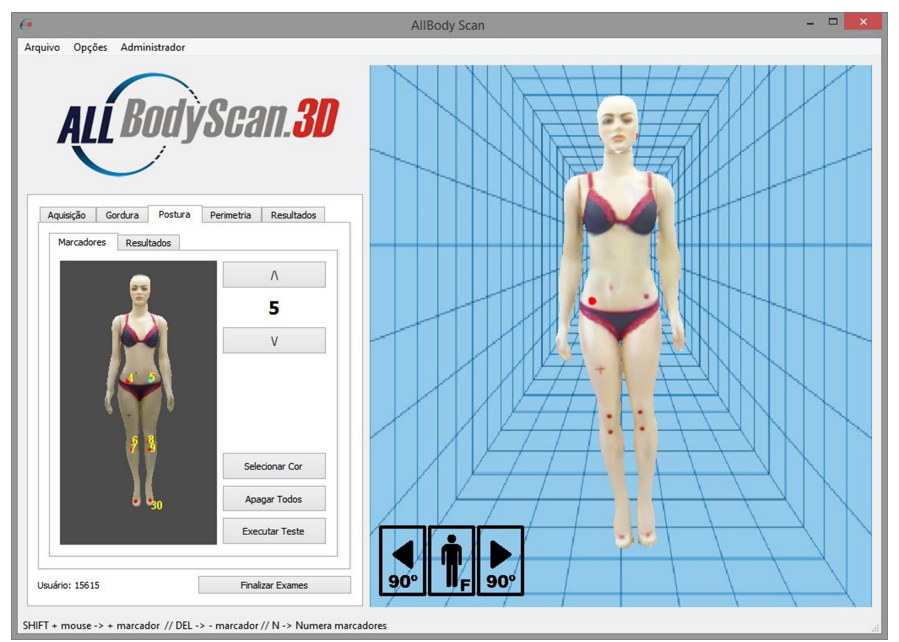

Figure 4. Interface of developed software and digital marking process.

The mesh volume is automatically calculated and the user inputs the weight (in kilograms) on the software and the body fat percentage is calculated.

The segmentation process is applied instantaneously and a vertical slider bar allows the user to select the height of the cutting plane. The perimeters of sections created at this height is calculated and exhibited on the screen, as shown in Figure 5.

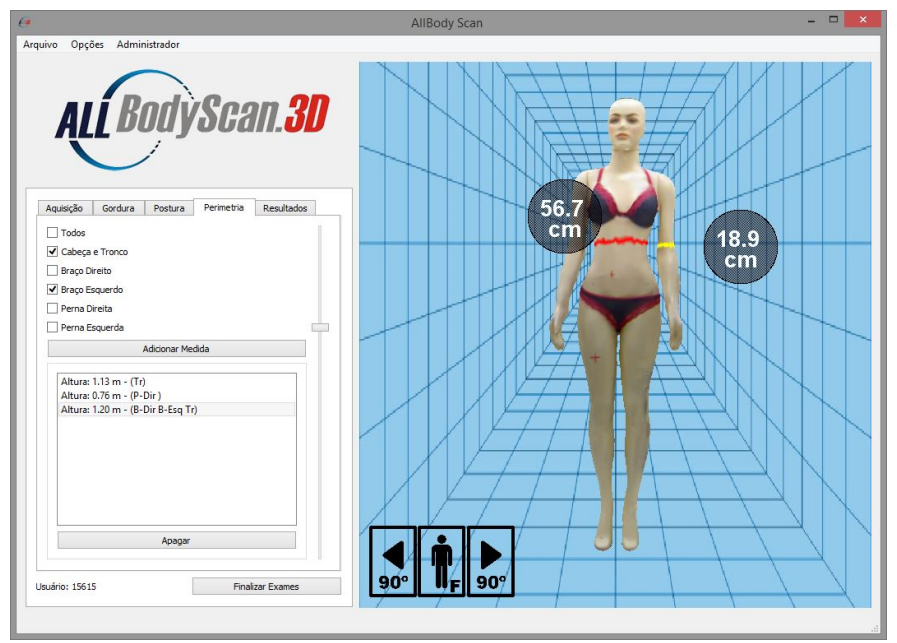

Figure 5. Measurement of body section perimeters.

All the results of the examinations can be exported to a PDF file in a single report. The posture analysis report section includes a series of images to illustrate all the numeric results. 
To test the posture analysis module, 10 tests on the developed software were compared to 10 tests using SAPO software. The analyzed subject was a real-size mannequin. The same meshes used on our software were used to generate the images for the SAPO input. The comparison showed good correspondence between the methods, as seen in Table 1.

Table 1. Results of comparison tests of postural analysis methods.

\begin{tabular}{|l||c|c|}
\hline & SAPO & AllBodyScan3D \\
\hline Exam & Mean Value & Mean Value \\
\hline Alignment - Head & $1.7^{\circ}$ & $1.6^{\circ}$ \\
Alignment - Acromion & $1.8^{\circ}$ & $2.0^{\circ}$ \\
Alignment - ASIS & $2.2^{\circ}$ & $1.9^{\circ}$ \\
Frontal Angle - Right Limb & $2.1^{\circ}$ & $2.1^{\circ}$ \\
Frontal Angle - Left Limb & $2.4^{\circ}$ & $2.5^{\circ}$ \\
Length Difference Between & $5 \mathrm{~mm}$ & $7 \mathrm{~mm}$ \\
Right and Left Limbs & & \\
Alignment - Tibias Tuberos- & $0.2^{\circ}$ & $0.1^{\circ}$ \\
ity - Head- & $43.2^{\circ}$ & \\
Right Hip Angle & $2.2^{\circ}$ & $2.1^{\circ}$ \\
Left Hip Angle & $10.3^{\circ}$ & $10.5^{\circ}$ \\
Alignment - Head-C7 & $45.0^{\circ}$ & $42.8^{\circ}$ \\
Alignment & $6.1^{\circ}$ & \\
Acromion & $1.1^{\circ}$ & $6.0^{\circ}$ \\
Alignment - Chest & $4.8^{\circ}$ & $1.2^{\circ}$ \\
Alignment - Hip & $15.8^{\circ}$ & $4.8^{\circ}$ \\
Alignment - Body & $4.5^{\circ}$ & $4.8^{\circ}$ \\
Alignment - Pelvis & $87.5^{\circ}$ & $84.9^{\circ}$ \\
Angle - Knee & $4.2^{\circ}$ & $4.0^{\circ}$ \\
Angle - Ankle & & \\
Angle Between Leg and & $6.7^{\circ}$ & $6.6^{\circ}$ \\
Foot Dorsum - Right & & \\
Angle Between Leg and & & \\
Foot Dorsum - Left & & \\
\hline
\end{tabular}

Calculations that took into account the markers of the inside of the foot presented difficulties in their reproduction due to the scanned model. The pose recommended for this type of examination requires a spacing between the legs of the user. In the scanned mannequin this space is very small, making it difficult to place the digital markers in this area.

The tests have shown that the proposed methodology has potential for use and may present improvements in the area of computerized postural analysis. The equipment is very suitable for the task and successfully created the 3D meshes of all users. The software, however, still requires further testing and clinical validation as well as distribution for experts in the field to use and to give a feedback about the ease of use and repeatability of results.

\section{Conclusion}

This paper presents a new methodology for postural analysis using three-dimensional scanned models in a way not yet seen in other works. The objective of constructing 
a multi-functional equipment for scanning and medical analysis was satisfactorily concluded, although it needs better visual treatment and aesthetic finish.

The developed software is able to reproduce the proposed methods and implement functionalities that require depth data such as mesh volume calculation for body composition analysis and perimeter measurement of body sections. The added features are not available in traditional computerized photogrammetry software.

The goal of creating an easy-to-use, intuitive system was fulfilled, resulting in a simple equipment and application requiring little user input. The scanning process is fully automatic, allowing even those who do not have any experience with 3D mesh processing to use the system.

The proposed method for the treatment of the mesh proved to be reliable. The scanning program was extensively used and showed no problems in the method. The problems presented in the generation of meshes were due to the user not staying in position and moving while the scanning was in progress, even when warned not to do so. The other problems with the scanning were presented in low light or direct sunlight ambient, which interferes with the measurement of infrared signals used by the sensors.

As this study focused on building the equipment and creating the software, the work still needs to be evaluated by experts of the field and needs to be validated in clinical tests and compared to other methods of measurement. However, tests performed with a human mannequin indicate that in a first analysis the system is ready for use with real patients. The comparison tests performed with the SAPO software showed a good matching of results. The evaluation process, however, still requires the user experience for the digital marking of the points in both systems, a factor that directly influences the repeatability of results.

\section{Acknowledgment}

This study was financed in part by the Coordenação de Aperfeiçoamento de Pessoal de Nível Superior - Brasil (CAPES) - Finance Code 001. The authors also thank professor Márcio Innocentini Guaratini, Paulo Suzuki and Jecel Mattos de Assumpção Jr. by review of the English.

\section{References}

Apeagyei, P. R. (2010). Application of 3d body scanning technology to human measurement for clothing fit.

Asanovic, I., Millward, H., and Lewis, A. (2019). Development of a 3d scan posturecorrection procedure to facilitate the direct-digital splinting approach. Virtual and Physical Prototyping, 14(1):92-103.

Berger, M., Tagliasacchi, A., Seversky, L., Alliez, P., Levine, J., Sharf, A., and Silva, C. (2014). State of the art in surface reconstruction from point clouds. In EUROGRAPHICS star reports, volume 1, pages 161-185.

Camelo, E. M. P. d. F., Uchôa, D. M., Uchoa Santos-Junior, F. F., Vasconcelos, T. B. d., and Macena, R. H. M. (2015). Uso de Softwares para Avaliação Postural: Revisão Integrativa. Coluna/Columna, 14:230 - 235. 
Chen, X., Golovinskiy, A., and Funkhouser, T. (2009). A benchmark for 3d mesh segmentation. In ACM Transactions on Graphics (TOG), volume 28, page 73. ACM.

Cignoni, P., Corsini, M., and Ranzuglia, G. (2008). Meshlab: An open-source 3d mesh processing system. Ercim news, 73(45-46):6.

Cui, Y., Chang, W., Nöll, T., and Stricker, D. (2012). Kinectavatar: Fully automatic body capture using a single kinect. In Asian Conference on Computer Vision, pages 133-147. Springer.

Danckaers, F., Huysmans, T., Hallemans, A., Bruyne, G. D., Truijen, S., and Sijbers, J. (2019). Posture normalisation of 3d body scans. Ergonomics, 0(0):1-15. PMID: 30777506 .

Delgado, L. (2004). Avaliação da composição corporal. São Luís.

Duarte, M., Ferreira, E. A., Maldonado, E. P., and Freitas, A. Z. Documentação sobre o SAPO - Software para Avaliação Postural.

Ferreira, E. A. G. (2005). Postura e Controle Postural: Desenvolvimento e Aplicação de Método Quantitativo de Avaliação Postural. PhD thesis, Universidade de São Paulo.

Han, H. and Nam, Y. (2011). Automatic body landmark identification for various body figures. International Journal of Industrial Ergonomics, 41(6):592-606.

Heindl, C., Bauer, H., Ankerl, M., and Pichler, A. (2015). ReconstructMe SDK: a C API for Real-time 3D Scanning. In 6th International Conference and Exhibition on 3D Body Scanning Technologies.

Kazhdan, M., Bolitho, M., and Hoppe, H. (2006). Poisson surface reconstruction. In Proceedings of the Fourth Eurographics Symposium on Geometry Processing, volume 7.

Lacerda, M. A. (2015). Aprimoramento do método de estimação de gordura corporal por meio de um scanner 3d antropométrico. Master's thesis, Universidade de São Paulo, São Carlos, SP.

Spahiu, T., Shehi, E., and Piperi, E. (2014). Extracting body dimensions from 3d body scanning. In 6th International Conference of Textile.

Tong, J., Zhou, J., Liu, L., Pan, Z., and Yan, H. (2012). Scanning 3d full human bodies using kinects. IEEE Transactions on Visualization and Computer Graphics, 18(4):643650 .

Zhong, Y. and Xu, B. (2006). Automatic segmenting and measurement on scanned human body. International Journal of Clothing Science and Technology, 18(1):19-30. 